\title{
Designing a linear pension scheme with forced savings and wage heterogeneity ${ }^{1}$
}

\author{
Helmuth Cremer ${ }^{2}$, Philippe De Donder ${ }^{3}$, \\ Dario Maldonado ${ }^{4}$ and Pierre Pestieau ${ }^{5}$
}

March 2006, revised January 2007

\footnotetext{
${ }^{1}$ We thank the referees and the editor, Jay Wilson for their detailed comments.

${ }^{2}$ University of Toulouse, GREMAQ and IDEI

${ }^{3}$ University of Toulouse, GREMAQ and IDEI.

${ }^{4}$ Universidad del Rosario, Bogota

${ }^{5}$ University of Liège, CORE, PSE and CEPR.
} 


\begin{abstract}
This paper studies the optimal linear pension scheme when society consists of rational and myopic individuals. Myopic individuals have, ex ante, a strong preference for the present even though, ex post, they would regret not to have saved enough. While rational and myopic persons share the same ex post intertemporal preferences, only the rational agents make their savings decisions according to these preferences. Individuals are also distinguished by their productivity. The social objective is "paternalistic": the utilitarian welfare function depends on ex post utilities. We examine how the presence of myopic individuals affects both the size of the pension system and the degree of redistribution it operates, with and without liquidity constraints. The relationship between proportion of myopic individuals and characteristics of the pension system turns out to be much more complex than one would have conjectured. Neither the impact on the level of pensions nor the effect on their redistributive degree are unambiguous. Nevertheless, we show that under some plausible assumptions adding myopic individuals increases the level of pension benefits and leads to a shift from a flat or even targeted scheme to a partially contributory one. However, we also provide an example where the degree of redistribution is not a monotonic function of the proportion of myopic individuals.
\end{abstract}

Keywords: social security, myopia, dual-self model

JEL classification: H55, D91 


\section{Introduction}

Public pension systems have three main functions. First, they force saving. Some individuals might be inclined to save less than the amount set aside through payroll taxes. Second, they redistribute income. Ideally, redistribution should be implemented by income taxation on a lifetime basis. Unfortunately, such a redistribution is rather limited in practice and, in most countries, pension systems are viewed as an effective and politically sustainable way of redistributing income in old age. ${ }^{1}$ Finally, public pensions provide insurance in a number of ways pertaining to health, longevity and financial risks. This paper is concerned with the first two functions, and particularly the first one. ${ }^{2}$

Even though the ideas of time inconsistency, myopia and procrastination and the ensuing need for forced saving have been present in the social security literature for decades, they have not been that popular in formal work. Redistributive considerations and concern for dynamic efficiency have dominated most of the theoretical work on social security. Feldstein (1985) is clearly an exception. His paper examines an overlapping generation economy with inelastic labor supply in which rational and myopic individuals coexist. $^{3}$ It analyses the welfare consequence of introducing a mandatory pension system and shows that the generosity of this system increases with the proportion of myopic agents. Feldstein's model was extended by Imrohoroglu et al. (2000) who introduce individual income risk, as well as mortality risks and borrowing constraints. They show that a pay-as-you-go pension system may raise welfare if the time inconsistency problem is large enough. There is also the recent paper by Diamond and Koszegi (2003) that is inspired by the hyperbolic discounting literature. Social security is there viewed as a commitment device.

The last decade has seen the emergence of behavioral economics which explores the

\footnotetext{
${ }^{1}$ In a comparison papier, we look at this issue of political sustainability. See Cremer et al. (2006b)

${ }^{2}$ There is another rationale for public pension that is not considered here and that appears when there is some sort of welfare or minimum pension. Some middle class workers could be tempted not to save at all and thus be entitled to such a pension benefit even though they can afford financing their retirement. By forcing them to save through the pension system, one solves what is known to be the "Samaritan dilemma". See on this, Pestieau and Possen (2006) and Homburg (2000).

${ }^{3}$ See also Feldstein (2002), sections 4.1 and 4.2 .
} 
possible conflict between our preferences for the long-run and our short-run behavior. The discrepancy between long-run intentions and short-run action is apparent for a wide range of circumstances and particularly for savings decisions. A number of surveys and experiments point out that a majority of people believe they should be saving more for retirement. ${ }^{4}$ This evidence suggests that households have self-control problems that call for commitment devices such as a public pension system. Quite interestingly, selfcontrol problems vindicate the idea of a paternalistic role for the government that for long was highly controversial.

In general, the self-control problem we have in mind is dealt with in the framework of hyperbolic preferences. In this paper, we adopt a two-period setting that does not lend itself to such preferences. Like Feldstein we consider a society in which two types of individuals coexist: rational ones who don't have to be forced to save and myopic ones who, ex ante, have a strong preference for the present even though, ex post, they would regret not to have saved enough. Like Imrohoroglu et al. (2000), we introduce explicit liquidity constraints, preventing young individuals from borrowing against their future pension benefits. Individuals are also distinguished by their productivity. The government has two objectives: forcing myopic individuals to save enough to support some basic standard of living throughout retirement and redistributing resources from high to low earners.

Designing an optimal pension system where all people are rational or myopic is pretty straightforward. The difficulty comes from mixing the two types and from mixing the objectives of forced saving and income redistribution.

We adopt a rather simple framework, namely a linear scheme with a payroll tax with uniform rate and pension benefits that have a contributory (Bismarckian) part and a flat rate (Beveridgean) part. ${ }^{5}$ To keep the model simple, we assume that the

\footnotetext{
${ }^{4}$ For a survey, see Angeletos et al. (2001).

${ }^{5}$ The nonlinear case is studied in a companion paper (Cremer et al. 2006a). Tenhunen and Tuomala (2006) also deal with nonlinear lifetime redistribution policies under myopia. Even though the nonlinear approach is in principle more general, it does not supersede the current paper. Nonlinear models provide interesting results in terms of marginal tax rates and distortions. However, discrete type models (the only tractable category in multideminsional taxation models) remain largerly agnostic regarding the properties of the implementing tax (and pension benefits) functions. Specifically, notions like generorsity and degree of redistribution do not have a clearcut interpretation in that setting. Tenhunen and Tuomala
} 
same distribution of productivity prevails in the two groups. The objective of the social planner is a utilitarian but paternalistic criterion. To be more precise, we consider the sum of individual utilities in which both rational and myopic individuals are given the same rate of time preference, namely that of the rational individuals. A main difference with the papers surveyed above is that the planner optimizes over both the generosity of pension system (measured by the contribution rate) and the link between individual pension benefit and past contribution (as measured by a parameter that we call the Bismarckian factor). Previous papers considered the second characteristic as exogenously given and concentrated on the contribution rate.

Our main results can be summarized as follows. Let us start with generosity and concentrate first on "homogeneous" societies (all rational or all myopic, but with individuals differing in productivity). When all individuals are rational the only justification of a public pension system is redistribution. The generosity of the system then depends on the wage inequality and on social preferences for equality. When all individuals are myopic, the main rationale for a pension system is to secure some level of resources in the retirement period and the generosity of the system is determined accordingly. Turning to "mixed" societies, the intuition would suggest that the generosity of the pension system should increase with the proportion of myopic individuals, in order to make up, at least in part, for their deficient private savings. This intuition is in line with the results derived by Feldstein (1985). He shows in a setting with exogenous labor supply where all workers have the same productivity that generosity unambiguously increases with the proportion of myopic individuals. In our setting this simple intuition no longer goes through. We find that increasing the proportion of myopics also has three other effects, all three pushing towards a less generous system. Consequently, the overall net impact is ambiguous. These three effects are linked to an increase in labor supply distortions and to a decrease in the importance of the redistributive motive as myopics get more numerous - two phenomenons that were not accounted for in Feldstein's setting.

Turning to the degree of redistribution, we ask if the pension system should be

(2006) measure redistribution by looking at Gini coeficients of first and second period consumption while Cremer et al. (2006a) focus on social welfare. 
Bismarckian, Beveridgean, or even targeted. With only rational individuals, a pure Beveridgean system is desirable when there are no binding liquidity constraints. When the liquidity constraints of some individuals are binding, targeting towards the poor is desirable (i.e., part of the pension benefits are decreasing with earlier income). With only myopic individuals, the Beveridgean formula always prevails. When the two types, rational and myopic households, are combined the pension system typically departs from the Beveridgean system, but in what direction is not obvious. We argue that targeting can emerge even in the absence of liquidity constraints (i.e., when the optimal system for the two groups taken separately is Beveridgean). However, liquidity constraints can also bring about targeting even when it is not otherwise optimal; this is shown through numerical examples.

\section{The model}

Rational individuals' utility is given by

$$
U_{R}=u(c-v(\ell))+u(d)
$$

where $c$ and $d$ are first- and second-period consumption, $\ell$ is first-period labor supply, $v(\ell)$ is the disutility from working and $u($.$) is the instantaneous utility from consumption,$ net of labor supply disutility. We assume that $u^{\prime}()>0,. u^{\prime \prime}()<0,. v^{\prime}(\ell)>0, v^{\prime \prime}(\ell)>0$. In the second period, individuals are retired. This utility (1) is also that of myopic individuals ex post. Ex ante, myopic agents totally forgo the second period and their utility is given by

$$
U_{M}=u(c-v(\ell)) .
$$

Individuals also differ in productivity $w \in\left[w_{-}, w_{+}\right]$. The distribution of $w$, represented by the distribution function $F($.$) , is independent of the proportions \pi_{R}$ and $\pi_{M}=1-\pi_{R}$ of rational and myopic individuals in the population. ${ }^{6}$

\footnotetext{
${ }^{6}$ In other words, the distribution of productivities is the same for the two types. This assumption is made to separate the impact of the proportion of myopics from the impact of the distribution of productivity. Other specification could be considered. If, for example, there were a strong positive correlation between myopia and productivity we would be in a world of non saving (where people are either too poor or too myopic to save) and the generosity of the pension system should be high.
} 
In the absence of a pension system, rational individuals choose $c, d$ and $\ell$ to maximize

$$
u(c-v(\ell))+u(d)
$$

We suppose a zero interest rate (and zero rate of population growth), so that $c+d=w \ell$.

For the myopic individuals, the problem is even simpler; they choose the value of $\ell$ that maximizes:

$$
u(w \ell-v(\ell))
$$

We now introduce a pension system consisting of a payroll tax $\tau$ and pension benefits $p$ that are given by

$$
p=\tau \alpha w \ell+\tau(1-\alpha) E w \ell .
$$

Here and throughout the paper the notation $E z$, where $z$ is any function of $w$, is used for its average value:

$$
E z=\int_{w_{-}}^{w_{+}} z(w) d F(w)
$$

The parameter $\alpha$ is often called the Bismarckian or the contributory factor. When $\alpha=0, p=\tau E w \ell$ and we have a flat benefit or (Beveridgean) pension system; all individuals receive the same pension irrespective of their contributions. When $\alpha=1$, $p=\tau w \ell$ and we have a purely contributory (Bismarckian) system; individuals' pensions are proportional to their contributions. If $\alpha<0$, pension benefits are inversely related to the wage level and we have a targeted pension system. In most countries, $\alpha$ is between 0 and 1.

The problem of rational individuals is given by

$$
\begin{array}{rl}
\max _{c, d, s, l} & u(c-v(\ell))+u(d), \\
\text { s.t. } & c+s \leq(1-\tau) w \ell, \\
& \quad d \leq s+\tau \alpha w \ell+\tau(1-\alpha) E w \ell .
\end{array}
$$

Let $x=c-v(\ell)$ denote the value of net consumption in period 1. We distinguish two cases depending on whether or not liquidity constraints are imposed.

Alternatively, if myopia and productivity were negatively related, myopia would not change much as it affects mainly low income earners are in any event likely to be liquidity constrained. 
When there are no liquidity constraints, $s$ can be negative and we have:

$$
\begin{aligned}
& u^{\prime}\left(x_{R}\right)=u^{\prime}\left(d_{R}\right), \\
& v^{\prime}\left(\ell_{R}\right)=(1-\tau(1-\alpha)) w .
\end{aligned}
$$

Equation (2) yields the labor supply function: $\ell_{R}=\ell(w(1-\tau(1-\alpha)))$. Labor supply increases with productivity $w$ and with the Bismarckian parameter $\alpha$, and decreases with the contribution rate $\tau$.

With liquidity constraints we have $s \geqslant 0 .{ }^{7}$ If $s>0$, we have the above first-order conditions. If, however, $(1-\tau) w l_{R}-v\left(\ell_{R}\right)<\tau \alpha w \ell_{R}+(1-\alpha) E w \ell, s=0$ and we have:

$$
\begin{aligned}
& u^{\prime}\left(x_{R}\right)>u^{\prime}\left(d_{R}\right), \\
& v^{\prime}\left(l_{R}\right)=(1-\tau) w+\frac{u^{\prime}\left(d_{R}\right)}{u^{\prime}\left(x_{R}\right)} \tau \alpha w,
\end{aligned}
$$

with

$$
\begin{aligned}
& x_{R}=(1-\tau) w \ell_{R}-v\left(\ell_{R}\right), \\
& d_{R}=\tau \alpha w \ell_{R}+(1-\alpha) \tau E w l .
\end{aligned}
$$

Consequently, labor supply depends on $w, \tau, \alpha$ for all rational individuals, and also on

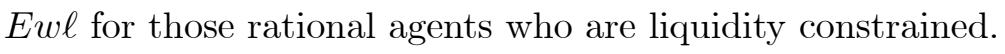

Turning to the myopic agent, his problem is simply to maximize

$$
u((1-\tau) w \ell-v(\ell))
$$

which yields

$$
v^{\prime}\left(\ell_{M}\right)=(1-\tau) w
$$

with

$$
\begin{aligned}
& x_{M}=(1-\tau) w l_{M}-v\left(l_{M}\right), \\
& d_{M}=\tau \alpha w l_{M}+(1-\alpha) \tau E w l .
\end{aligned}
$$

\footnotetext{
${ }^{7}$ Assuming liquidity constraints with mandatory (private or public) pensions is both standard and realistic. It is typically impossible to borrow against the promise of future public pension payments. Even in the case of private contributive but mandatory schemes, it is generally acknowledged that liquidity constraints apply (so that contributions to those scheme are distortionary). See on this Summers (1989).
} 
We thus obtain $\ell_{M}=\ell(w(1-\tau))$.

Note that if $\alpha=0$, labor supply is identical in each of the three cases, as it depends only on $w(1-\tau)$. This is due to the fact that we have assumed away income effects. We shall distinguish cases according to whether or not savings can be negative. When there is no liquidity constraint, savings can be negative. Saving might also be positive for all rational agents, which is the case when pension benefits are low because, e.g., distortions are very high. When savings are strictly positive for all rational agents, we have $u^{\prime}\left(x_{R}\right)=u^{\prime}\left(d_{R}\right)$. Furthermore, given our assumptions we have $u^{\prime}\left(x_{M}\right)<u^{\prime}\left(d_{M}\right)$ for all myopic individuals. In other words, all myopic individuals, even the poor, consume more in the first than in the second period. We use this result below.

\section{Government's problem}

We now consider the problem of a social planner dealing with a society of rational and myopic individuals who also differ productivities. Its objective is the sum of ex post individual utilities, represented by the same utility functions for rational and myopic individuals. The idea is that ex post the myopic will be grateful to their government for having forced them to save.

Two words on this specification. First, the use of a utilitarian welfare function is standard. As an alternative we could have used a Rawlsian criterion which would consist in maximizing the welfare of the myopic and least productive individuals. Second, we adopt here an approach that is now called "new paternalism". According to this view, paternalistic intervention can be justified to correct problems of self-control. ${ }^{8}$

Using our compact notation, the problem of the social planner is expressed as follows:

$$
\begin{aligned}
\mathcal{L}= & \sum_{j=M, R} \pi_{j}\left\{E u\left[w(1-\tau) \ell_{j}-s_{j}-v\left(\ell_{j}\right)\right]\right. \\
& \left.+E u\left[s_{j}+\tau\left(\alpha w \ell_{j}+(1-\alpha) E w \ell\right)\right]\right\}
\end{aligned}
$$

\footnotetext{
${ }^{8}$ See on this Benabou and Tirole (2002).
} 
Differentiating this expression yields:

$$
\begin{aligned}
\frac{\partial \mathcal{L}}{\partial \tau}= & -\sum \pi_{j}\left\{E\left[w \ell_{j}\left(u^{\prime}\left(x_{j}\right)-\alpha u^{\prime}\left(d_{j}\right)\right)-u^{\prime}\left(d_{j}\right)(1-\alpha) E w \ell\right]\right\} \\
& +\pi_{M} \tau \alpha E\left[w \frac{\partial \ell_{M}}{\partial \tau} u^{\prime}\left(d_{M}\right)\right]+\sum \pi_{j} E u^{\prime}\left(d_{j}\right) \tau(1-\alpha) E w \frac{\partial \ell_{j}}{\partial \tau} \\
\frac{\partial \mathcal{L}}{\partial \alpha}= & \sum \pi_{j}\left\{\tau \operatorname{cov}\left(w \ell_{j}, u^{\prime}\left(d_{j}\right)\right)+(1-\alpha) \tau E u^{\prime}\left(d_{j}\right) E\left(w \frac{\partial \ell_{j}}{\partial \alpha}\right)\right\} .
\end{aligned}
$$

Setting these expressions equal to zero yields a system of two equations that jointly determine the optimal levels of $\tau$ and $\alpha$ (as long as the solutions is interior). ${ }^{9}$ While generosity and the degree of redistribution are jointly determined it is interesting for expositional reasons to discuss these issues separately. ${ }^{10}$ Doing this, we do of course have to keep in mind that the two variable are not independent.

\subsection{Optimal tax rate}

We first focus on the optimal tax rate for a given $\alpha$. Rearranging (4) we obtain,

$$
\begin{aligned}
\frac{\partial \mathcal{L}}{\partial \tau}= & -\sum \pi_{j}\left\{(1-\alpha) \operatorname{cov}\left[w \ell_{j}, u^{\prime}\left(d_{j}\right)\right]-E\left[w \ell_{j}\left(u^{\prime}\left(d_{j}\right)-u^{\prime}\left(x_{j}\right)\right)\right]\right\} \\
& +\sum \pi_{j}(1-\alpha) \tau E\left[u^{\prime}\left(d_{j}\right)\right] E\left[w \frac{\partial \ell_{j}}{\partial \tau}\right]+\pi_{M} \tau \alpha E\left[w \frac{\partial \ell_{M}}{\partial \tau} u^{\prime}\left(d_{M}\right)\right] .
\end{aligned}
$$

Setting this expression equal to zero and solving yields:

$$
\tau=\frac{\sum \pi_{j}\left[(1-\alpha) \operatorname{cov}\left(w \ell_{j}, u^{\prime}\left(d_{j}\right)\right)\right]-\sum \pi_{j} E\left[w \ell_{j}\left(u^{\prime}\left(d_{j}\right)-u^{\prime}\left(x_{j}\right)\right)\right]}{\sum \pi_{j}(1-\alpha) E u^{\prime}\left(d_{j}\right) E\left(w \frac{\partial \ell_{j}}{\partial \tau}\right)+\pi_{M} \alpha E\left(w \frac{\partial \ell_{M}}{\partial \tau} u^{\prime}\left(d_{M}\right)\right)} .
$$

The two terms in the denominator reflect the efficiency concern and depend on the tax elasticity of labor supply, which is negative. The first term, which relates to the non contributory part of the pension scheme for all agents, is standard. The second term focuses on the contributory part for the myopic individuals only, since unlike rational agents they fail to factor in the link between pensions and contributions when choosing how much labor to supply. The two terms that compose the numerator reflect the equity concerns. The covariance term represents the redistributive objective: this term

\footnotetext{
${ }^{9}$ Keeping in mind of course that the individual decision variables $\ell_{j}$ and $d_{j}$ are functions of $\tau$ and $\alpha$ as discussed in Section 2.

${ }^{10}$ Given the complexity of the problem, a more direct comparative statics approach based on the sytem (4)-(5) is not very insightful in itself.
} 
is usually negative as the level of earnings and the marginal utility of second period consumption are negatively correlated. The second term, which we call consumption smoothing, comes from the desire to secure enough consumption for all individuals in both periods. To get a better grasp at its impact, assume that we are in a case where saving is positive for all rational agents. In that case, $d_{M}<x_{M}$ for all myopic agents, so that the consumption smoothing term calls for a higher tax rate in order to decrease the consumption gap between the two periods for the myopic individuals. On the other hand, if the non negative saving constraint is binding for some rational agents, the consumption smoothing term for them would call for a lower payroll tax rate in order to increase their first period consumption.

Before turning to the impact of having myopic individuals on the optimal tax rate, we briefly discuss how the optimal payroll tax rule specified by (7) is affected by the labor supply elasticity and by the degree of concavity of the utility function. ${ }^{11}$ Labor supply elasticity only influences the denominator of (7). A lower sensitivity of labor supply to the net return of working decreases the (absolute value of) denominator and leads to a higher contribution rate, as is intuitive. ${ }^{12}$ As for the concavity of the utility function, it mainly influences the covariance term in the numerator representing the redistributive objective. To see this, assume a constant marginal utility of consumption. In that case, the covariance term disappears, annihilating the redistributive motive. We then obtain that a less concave utility function calls for a lower optimal contribution rate. ${ }^{13}$

Equation (7) shows that the impact on the optimal contribution rate of having myopic individuals in society is complex. One can identify four effects. First, the difference between first and second period consumption is larger for myopic than for rational agents of same productivity, so that the consumption smoothing term calls for a higher payroll tax. This effect corresponds to the intuition that the payroll tax should

\footnotetext{
${ }^{11}$ This discussion, like most of the other arguments in Subsections 3.1 and 3.2, are very much in the optimal linear income tax tradition, and subject to the usual caveats. Specifically, our conclusions mostly pertain to tax rules. Conclusions about the actual levels are typically not available at a high level of generality. We, however, provide illustrative results about levels in Section 4.

${ }^{12}$ Because of the absence of income effects in our setting, the derivatives that appear in the denominator of (7) concern the compensated labor supply functions.

${ }^{13}$ The optimal contribution tax rate is also affected by changes in wage inequality, but in a less straightforward manner.
} 
be higher with myopic individuals to compensate for their second period consumption being too low. However, the other three effects go in the opposite direction. Myopic individuals save less than rational agents, which tends to reduce the absolute value of the (negative) average covariance in the numerator of (7); this calls for a lower $\tau$. The third effect goes in the same direction: with myopic individuals, the contributory part of the pension scheme generates labor supply distortions, which in turn call for a lower payroll tax. ${ }^{14}$ Finally, the marginal utility of second period consumption is larger for myopic than for rational agents of same productivity, which increases the distortionary impact of the Beveridgean part of the pension scheme and also calls for a lower payroll tax. The net effect of the presence of myopic individuals can of course not be assessed by counting the terms that go one way or the other. The crucial factor is their magnitude which cannot be assessed at this level of generality. The numerical examples given below show that the initial intuition may well go through in spite of the presence of these countervailing effects.

When we compare the extreme settings of $\pi_{M}=0$ (no myopic individuals) and $\pi_{M}=1$ (all myopic society) the expression is simplified but remains ambiguous. To see this most clearly we make two additional simplifying assumptions, namely $s_{R}>0$ and $\alpha=0$. The assumption that $s_{R}>0$ means that no rational individual faces a (binding) liquidity constraint, which translates in a consumption smoothing term that is nil for rational agents and positive for myopic individuals. The assumption that $\alpha=0$ means that we concentrate on a Beveridgean system, under which the distortion generated by the contributory part of the pension system on myopic individuals' labor supply disappears..$^{15}$

The optimal payroll tax with rational agents only is then given by:

$$
\tau^{R}=\frac{-\operatorname{cov}\left(w \ell_{R}, u^{\prime}\left(d_{R}\right)\right)}{-E u^{\prime}\left(d_{R}\right) E\left(w \frac{\partial \ell_{R}}{\partial \tau}\right)} .
$$

\footnotetext{
${ }^{14}$ Recall that the contributory part of the pension scheme does not generate distortions for rational individuals because they anticipate the induced increase in pensions.

${ }^{15}$ In the next subsection we will show that in the absence of liquidity constraints $\alpha=0$ is effectively optimal for $\pi_{M}=0$ and $\pi_{M}=1$.
} 
With myopic individuals only we have

$$
\tau^{M}=\frac{-E\left[w \ell_{M}\left(u^{\prime}\left(d_{M}\right)-u^{\prime}\left(x_{M}\right)\right)\right]}{u^{\prime}\left(d_{M}\right) E\left(w \frac{\partial \ell_{M}}{\partial \tau}\right)},
$$

Comparing to the general expression (7), we can notice that the numerator of the expression for $\tau^{R}$ does not contain the consumption smoothing term while the numerator of $\tau^{M}$ has no covariance term (with $\alpha=0, d_{M}$ is the same for all so that $\left.\operatorname{cov}\left(w \ell_{M}, u^{\prime}\left(d_{M}\right)\right)=0\right)$. In spite of these simplifications the comparisons between the numerators in the expression for $\tau^{R}$ and $\tau^{M}$ remains ambiguous. The denominator, on the other hand can be expected to be larger with myopic individuals. This corresponds to the fourth effect identified in the discussion of the general expression.

Summing up, the net effect of the presence of myopic individuals on the optimal tax rate appears to be ambiguous. The numerical examples presented below suggest that the consumption smoothing term appears to dominate in a wide range of settings. However, our examples are purely illustrative; the precise comparison remains an empirical question that requires at the very least simulations based on a calibrated version of the model.

\subsection{The optimal value of the Bismarckian factor}

Using $\partial \ell_{M} / \partial \alpha=0$, the first-order condition with respect to $\alpha$, (5), can be rewritten as

$$
\frac{\partial \mathcal{L}}{\partial \alpha}=\sum \pi_{j}\left\{\tau \operatorname{cov}\left(w \ell_{j}, u^{\prime}\left(d_{j}\right)\right)\right\}+\pi_{R} \tau(1-\alpha) \tau E u^{\prime}\left(d_{R}\right) E\left(w \frac{\partial \ell_{R}}{\partial \alpha}\right)
$$

This condition trades off the anti-redistributive impact of $\alpha$ (which increases consumption inequality, as measured by the first term on the RHS) with its efficiency-enhancing effect on the labor supply of rational individuals (labor supply of myopics is not affected by $\alpha) .{ }^{16}$ Here also, the impact of modifying labor supply elasticity and the concavity of the utility function is clear. Decreasing labor supply elasticity dampens the efficiencyenhancing effect of linking pension benefits with individual contributions and thus calls

\footnotetext{
${ }^{16}$ We can also use $(1-\alpha)$ as decision variable. Noting that $(1-\alpha) \tau$ represents the net payroll tax rate, (10) can be interpreted as a traditional linear income tax rule.
} 
for a lower value of $\alpha$. Increasing the concavity of the utility function reinforces the equity concern and also calls for a lower value of $\alpha$.

To better understand the implications of the proportion of myopic citizens for the determination of the optimal $\alpha$, we cover successively the cases where society is composed of myopic agents only, of rational agents only, and of both types of agents.

When society is composed only of myopic individuals, equation (10) simplifies to

$$
\frac{\partial \mathcal{L}}{\partial \alpha}=\tau \operatorname{cov}\left(w \ell_{M}, u^{\prime}\left(d_{M}\right)\right)
$$

which is zero when $\alpha=0$ : with a flat pension $d_{M}=p$ is constant for all $w$ so that the covariance is equal to zero. Furthermore, when $\alpha>0$ the covariance is negative, while it is positive when $\alpha<0$. Consequently, the optimal level of $\alpha$, denoted by $\alpha^{M}$ is zero. ${ }^{17}$ Intuitively, with only myopic agents there is no (efficiency) reason to link pension benefits to contributions, and the social planner's redistributive objective leads to the adoption of a Beveridgean scheme.

We now look at a society composed exclusively of rational agents. Setting (6) equal to zero and substituting in (10) yields

$$
\begin{aligned}
\frac{\partial \mathcal{L}}{\partial \alpha}= & \frac{1}{1-\alpha} E w \ell_{R}\left(u^{\prime}\left(d_{R}\right)-u^{\prime}\left(x_{R}\right)\right) \\
& +E u^{\prime}\left(d_{R}\right) E\left[w\left(\frac{\partial \ell_{R}}{\partial \tau} \tau+\frac{\partial \ell_{R}}{\partial \alpha}(1-\alpha)\right)\right] .
\end{aligned}
$$

In the absence of liquidity constraints (or with $s_{R}>0$ ) the first term in (11) vanishes. Observe that, by equation (2), the labor supply of non liquidity constrained rational individuals is a function of $\tau(1-\alpha)$. It follows that the second term in (11) vanishes also. Furthermore, only the optimal value of $\tau^{R}\left(1-\alpha^{R}\right)$ matters and thus there is no single optimal value of $\alpha^{R}$. To be more precise, the optimal combinations of $\tau^{R}$ and $\alpha^{R}$ are defined by the equation $\tau^{R}\left(1-\alpha^{R}\right)=\tau^{*}$, where $\tau^{*}$ is the optimal tax rate conditional on $\alpha=0 .{ }^{18}$ Intuitively, with non liquidity constrained rational agents, the payroll tax and the Bismarckian parameter are perfect substitutes. Their optimal combinations trade off redistribution and the labor supply distortions generated by the non contributory part of the pension system.

\footnotetext{
${ }^{17}$ This argument is valid for any level of $\tau>0$, including the optimal one.

${ }^{18}$ One can also interpret $\tau^{*}$ as the optimal net payroll tax rate.
} 
We now assume that some rational individuals are liquidity constrained. For those individuals (low-wage earners), $d_{R}>x_{R}$ and

$$
\frac{\partial \ell_{R}}{\partial \tau} \tau+\frac{\partial \ell_{R}}{\partial \alpha}(1-\alpha)<0
$$

by virtue of (3), so that both terms in (11) are negative. Put differently, the perfect substitutability between $\tau$ and $\alpha$ then disappears when some rational agents are liquidity constrained, and the optimal value of $\alpha$ becomes negative. The intuition for this result is that introducing targeting into the pension system (by means of a negative value of $\alpha$ ) has nice redistributive properties while being less damaging to efficiency than in the no liquidity constraint case, since the sensitivity of labor supply to variations of $\alpha$ is dampened by the lower relative utility of second period consumption.

Finally, when both myopic and rational agents coexist, one can show that

$$
\left.\frac{\partial \mathcal{L}}{\partial \alpha}\right|_{\alpha=0}=\pi_{R} \tau \operatorname{cov}\left(w \ell_{R}, u^{\prime}\left(d_{R}\right)\right)+\pi_{R} \tau E u^{\prime}\left(d_{R}\right) E w \frac{\partial \ell_{R}}{\partial \alpha} .
$$

The first term of the right hand side is negative but the second one is positive and thus we cannot sign this expression. Interestingly, this ambiguity arises in the absence of liquidity constraints. Recall that without liquidity constraints $\alpha=0$ is optimal when society is "homogenous" (only rational or only myopic individuals). However, in a mixed society, the optimal level of $\alpha$ will in general differ from zero. Specifically, when labor supply elasticity is small $\partial \ell_{R} / \partial \alpha \rightarrow 0$, the second term on the RHS of (12) vanishes and we obtain a negative level of $\alpha$. In other words, in the absence of labor market distortions a very redistributive system is optimal. On the other hand, if the degree of concavity of $u$ is small ( $u$ is close to a linear function), the first term become negligible and we have $\alpha>0$. In that case, redistributive benefits are small and we want to make the system more Bismarckian to mitigate labor market distortions.

To sum up the main conclusions of this section, we show that $\tau^{M}$ is likely to be larger than $\tau^{R}$ particularly when the rational individuals are not liquidity constrained. We also show that $\alpha^{R}$ is determined by $\tau^{R}\left(1-\alpha^{R}\right)=\tau^{*}$ when rational agents are not liquidity constrained. When they are, $\alpha^{R}$ can be negative. As to $\alpha^{M}$ it is zero. The sign of the optimal value of $\alpha$ is ambiguous when both rational and myopic individuals coexist. Table 1 summarizes the results pertaining to the optimal value of $\alpha$. 


\begin{tabular}{ccc}
\hline$\pi^{R}$ & No (binding) liquidity constraints & Binding liquidity constraints \\
\hline 0 & $\alpha=0$ & $\alpha=0$ \\
$0<\pi^{R}<1$ & $\alpha \lesseqgtr 0$ & $\alpha \lesseqgtr 0$ \\
1 & $\tau(1-\alpha)=\tau^{*}$ & $\alpha<0$ \\
\hline
\end{tabular}

Table 1: Summary of Theoretical Results: Optimal Value of $\alpha$.

In view of such an ambiguity, we now turn to a simulation exercise. This example not only illustrates our findings but also provides some extra results pertaining to the actual levels of $\tau$ and $\alpha$ that occur in the different case. While these results are not general, they are instructive in that the show that some patterns of results are effectively possible.

\section{Numerical example}

Our numerical simulations are based on the following utility function,

$$
u=\log \left(c-\ell^{2} / 2\right)+\log d
$$

and a positively skewed $\operatorname{Beta}(2,4)$ distribution for the wages with support $(1,4)$. Table 2 presents the optimal value of $\alpha$ and $\tau$ for alternative values of the proportion of rational individuals, $\pi^{R}$. It also gives the optimal values of $\tau$ when $\alpha=0$, which we denote by $\tau^{*}$. In calculating these values, we assume away any liquidity constraint so that the poor rational individuals exhibit negative savings. Table 3 presents the same results for the case where liquidity constraints are imposed.

We start with Table 2. We know from theory that the optimal value of $\alpha$ is zero in the absence of rational individuals, and that $\tau$ and $\alpha$ are perfectly substitutable when society is composed only of non liquidity constrained individuals. The optimal value of $\tau(1-\alpha)$ when $\pi^{R}=1$ is given by $\tau^{*}$. The comparison between the optimal Beveridgean payroll tax when there are only rational individuals who save and when there are only myopic individuals is in general ambiguous, although we conjectured that it would be larger with myopic individuals. This conjecture is borne out by the results reported in Table $2 .{ }^{19}$

\footnotetext{
${ }^{19}$ The situation we simulate in Table 2 is slightly different, since even though all rational agents
} 


\begin{tabular}{cccc}
\hline$\pi^{R}$ & $\alpha$ & $\tau$ & $\tau^{*}$ \\
\hline 0.0000 & 0.000 & 0.250 & 0.250 \\
0.1000 & 0.066 & 0.247 & 0.247 \\
0.2000 & 0.122 & 0.245 & 0.243 \\
0.4000 & 0.209 & 0.242 & 0.233 \\
0.6000 & 0.272 & 0.239 & 0.220 \\
0.7000 & 0.298 & 0.238 & 0.211 \\
0.9000 & 0.342 & 0.236 & 0.209 \\
0.9300 & 0.347 & 0.236 & 0.239 \\
0.9600 & 0.353 & 0.236 & 0.220 \\
0.9900 & 0.358 & 0.235 & 0.209 \\
0.9990 & 0.360 & 0.235 & 0.151 \\
0.9999 & 0.360 & 0.235 & 0.151 \\
1.0000 & - & - & 0.151 \\
\hline
\end{tabular}

Table 2: Optimal linear pension scheme as a function of the proportion of rational individuals. No liquidity constraint. We denote $\tau^{*}$ the optimal tax rate for $\alpha=0$.

Recall that the optimal payroll tax rate trades off four concerns, one of them in favor of a larger tax (consumption smoothing) while three of them plead for a lower tax (redistributive concern as well as labor supply effects of the non contributory part of pension for all individuals, and of the contributory part for myopic agents). The presence of myopic agents tends to increase the consumption smoothing effect. Assuming that the pension system is Beveridgean $(\alpha=0)$, it seems reasonable to surmise that the optimal payroll tax increases with the proportion of myopic agents, which is the result we obtain in Table 2 by looking at the $\tau^{*}$ column. Moreover, the optimal value of $\tau$ (obtained by optimizing simultaneously over $\tau$ and $\alpha$ ) is also increasing with the proportion of myopic individuals.

The optimum value of $\alpha$ trades off redistribution (calling for moving away from a Bismarckian system towards a Beveridgean or even a targeted system) and efficiency (calling for a tighter link between pension benefits and contributions). Since a Bismarckian system fares no better, in efficiency terms, than a Beveridgean one for myopic

equalize marginal utility in both periods, the lowest earners do so by borrowing rather than saving. This means that, although the consumption smoothing term $u^{\prime}\left(d_{R}\right)-u^{\prime}\left(x_{R}\right)$ in $(7)$ is nil for all rational agents, it is negative for low productivity myopic agents and positive for the other myopic individuals. It is nevertheless reasonable to assume that, on average, the consumption smoothing term is positive for myopic agents. 


\begin{tabular}{cccc}
\hline$\pi^{R}$ & $\alpha$ & $\tau$ & $\tau^{*}$ \\
\hline 0.0000 & 0.000 & 0.250 & 0.250 \\
0.1000 & 0.072 & 0.246 & 0.245 \\
0.2000 & 0.131 & 0.243 & 0.240 \\
0.4000 & 0.220 & 0.236 & 0.228 \\
0.6000 & 0.277 & 0.230 & 0.213 \\
0.7000 & 0.295 & 0.225 & 0.203 \\
0.9000 & 0.290 & 0.209 & 0.191 \\
0.9300 & 0.269 & 0.202 & 0.168 \\
0.9600 & 0.232 & 0.192 & 0.160 \\
0.9900 & 0.106 & 0.166 & 0.151 \\
0.9990 & -0.184 & 0.127 & 0.148 \\
0.9999 & -0.346 & 0.111 & 0.147 \\
1.0000 & -1.360 & 0.064 & 0.147 \\
\hline
\end{tabular}

Table 3: Optimal linear pension scheme as a function of the proportion of rational individuals. Liquidity constraint

individuals, intuition suggests that the optimal system would be more and more Beveridgean as the proportion of myopic individuals increases, i.e., that the optimal value of $\alpha$ should increase with $\pi^{R}$. This is what we obtain in Table 2 .

We now turn to Table 3 . We know from theory that the optimal value of $\alpha$ is zero in the absence of rational individuals, and is negative when society is composed only of rational individuals, some of them being liquidity constrained. Comparison of Tables 2 and 3 shows that when all individuals are rational the optimal value of $\tau(1-\alpha)$ is smaller when a liquidity constraint is imposed on the rational individuals than when there is no liquidity constraint. This is because when there is a liquidity constraint the higher tax has a more significant effect on the first period consumption of the poor (who benefit from the redistribution, but only in the second period). When all individual are myopic, on the other hand, the liquidity constraint imposed on the rational individuals is of course irrelevant; this explains why the level of $\alpha^{*}$ for $\pi^{R}=0$ is the same in both tables. We also obtain from Table 3 that the optimal payroll tax when $\alpha=0$ is larger when there are only rational individuals than when there are only myopic individuals. In other words, the fact that some rational agents are credit constrained and exhibit a negative consumption smoothing term is not enough to reverse the relationship we 


\begin{tabular}{ccc}
\hline$\pi^{R}$ & No (binding) liquidity constraints & Binding liquidity constraints \\
\hline 0 & 0 & 0 \\
$0<\pi^{R}<1$ & $>0$ & $>0$ for low values of $\pi^{R}$ \\
& & $<0$ for $\pi^{R}$ close to 1 \\
1 & $\tau(1-\alpha)=0.151$ & $<0$ \\
\hline
\end{tabular}

Table 4: Summary of Numerical Results: Optimal Value of $\alpha$.

obtain from theory when all rational agents exhibit positive savings. On the other hand, by comparing the last columns of Tables 2 and 3, it is clear that this consumption smoothing effect leads to a lower optimal tax rate (conditional on $\alpha=0$ ), when credit constraints are imposed than when they are not.

We also obtain, in Table 3 as in Table 2, that the optimal payroll tax rate (both when $\alpha$ is set at zero and when it is also optimized) decreases monotonically with $\pi^{R}$. The main difference between the two Tables lies in the behavior of the optimal $\alpha$ with respect to the proportion of myopic agents. When some rational individuals are liquidity constrained, the optimal $\alpha$ first rises then decreases with $\pi^{R}$. The largest optimal non contributory part of the pension system is obtained when $30 \%$ of the population consists of myopic individuals, and decreases as one moves away from that proportion.

Table 4 summarizes our numerical results regarding the optimal value of $\alpha$.

\section{Conclusion}

In this paper, we have analyzed the linear pension schemes that ought to be applied in a society wherein myopic and rational agents coexist and the government forces the former to save. The foundation of such a paternalistic behavior lies in the fact that myopic individuals will be grateful in the second period of their life to the government for having forced them to save for their old age. In other words, myopic agents have two selves, one looking for immediate gratification and one looking for long term welfare in the tradition of "dual-self" models (see, e.g., O'Donoghue and Rabin (2001)).

We show that introducing that type of agents in a society of rational individuals has implications for the level of pension benefits and the redistributiveness of the system 
that are far more complex than one would have expected. Intuitively one expects that adding myopic individuals increases the level of pension benefits and leads to a less redistributive system. We show that this intuition goes through under some conditions. Specifically, the presence of myopic individuals may even induce a shift from a targeted scheme to a partially contributory one. However, no definitive results can be obtained in a general setting and the assessment of net effect appears to be an empirical question.

To keep the analysis simple, we have restricted the pension system to a linear scheme and we have assumed that the rate of return and the rate of population growth were not only the same but equal to zero. Relaxing the latter assumption would not change the qualitative nature of our results. Allowing for a non linear scheme is likely to have strong implications depending on informational assumptions, as we show in a companion paper (Cremer et al. (2006a)).

This paper is normative; a paternalistic social planner designs an optimal pension system, optimal from an ex post standpoint for the myopic individuals. In Cremer et al. (2006b), we have also analyzed the political economy aspect of the same problem wherein individuals, myopic and rational, vote ex ante for a pension system. We there assume that the myopic, when they vote, are in a kind of "state of grace", which makes them choose a commitment device. Interestingly, we obtain results that are parallel to those found in this paper: the introduction of myopic agents increases the generosity and decreases the redistributiveness of the pension system.

\section{References}

[1] Angeletos, G., D. Laibson, A. Repetto, J. Tobacman and S. Weinberg, (2001), The hyperbolic consumption model: calibration, simulation and empirical evaluation, Journal of Economic Perspectives, 15, 47-68.

[2] Benabou, R. and J. Tirole, (2002), Self-confidence and personal motivation, The Quarterly Journal of Economics, 117:3, 871-915.

[3] Cremer, H., Ph. De Donder, D. Maldonado and P. Pestieau, (2006a), Forced saving, redistribution and non linear social security scheme, unpublished. 
[4] Cremer, H., Ph. De Donder, D. Maldonado and P. Pestieau, (2006b), Voting over type and generosity of a pension system when some individuals are myopic, CORE DP 2006/79.

[5] Diamond, P. and B. Koszegi, (2003), Quasi-hyperbolic discounting and retirement, Journal of Public Economics, 87, 1839-1872.

[6] Feldstein, M., (1985), The optimal level of social security benefits, Quarterly Journal of Economics, 100, 303-321.

[7] Feldstein, M., (2002), Social security, in Handbook of Public Economics, vol. 4, ed. by A. Auerbach and M. Feldstein, North Holland, Amsterdam, 2246-2324.

[8] Homburg, S., (2000), Compensatory saving in the welfare state, Journal of Public Economics, 77, 233-239.

[9] Imrohoroglu, A., S. Imrohoroglu and D. Joines, (2003), Time inconsistent preferences and social security, Quarterly Journal of Economics, 118-2, 745-784.

[10] O'Donoghue, T. and M. Rabin, (2001), Choice and procrastination, Quarterly Journal of Economics, 116, 121-160.

[11] Pestieau, P. and U. Possen, (2006), Prodigality and myopia. Two rationales for social security, CORE DP 2006/73.

[12] Summers, L., (1989), Some simple economics of mandated benefits, American Economic Review, 79, 177-183.

[13] Tenhunen, S. and M. Tuomala, (2006), On optimal lifetime redistribution policy, mimeo University of Tampere. 